\title{
IS THERE A FUNDAMENTAL DIFFERENCE BETWEEN A LIQUID AND A GAS?
}

\author{
D. H. DOUGLASS, Jr. * \\ Department of Physics and Institute for the Study of Metals \\ The University of Chicago \\ (Received 1 April 1965)
}

\begin{abstract}
Operationally, an extension of the liquid-gas equilibrium curve above the classical critical point can be defined. The existence of such a line may mean that there is a fundamental difference between a liquid and a gas.
\end{abstract}

THE ACCEPTED view is that a liquid and a gas differ only in the values of their extensive variables such as the volume and entropy. It is often stated that one can not tell whether a fluid contained in a vessel is a liquid or a gas if there is not some of the other phase present. This view further states that there is a critical temperature and pressure above which there exists only a single phase with the concepts of gas phase and liquid phase losing their meaning.

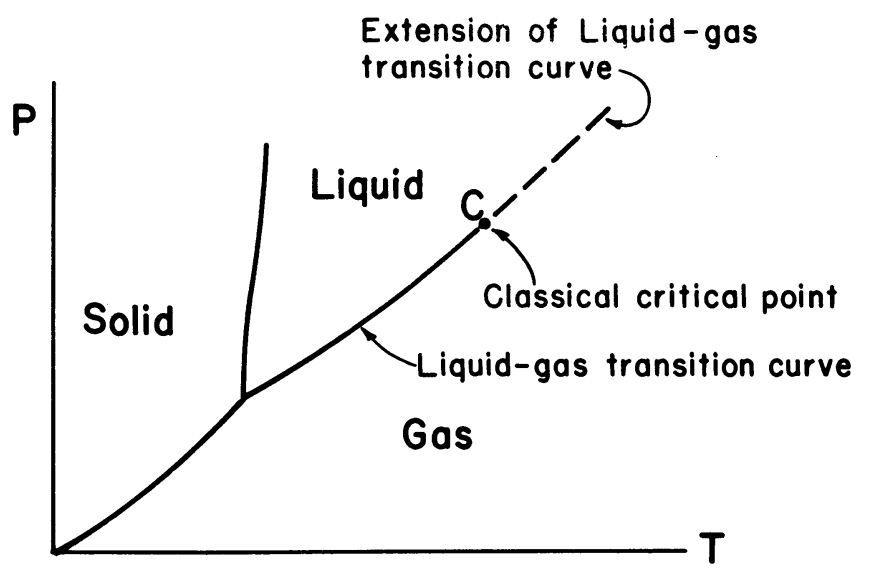

FIGURE 1

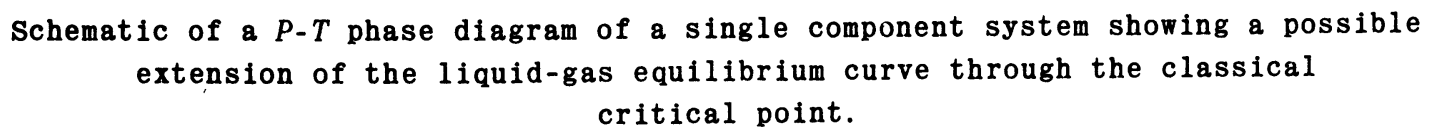

We wish in this paper to consider the possibility that there is a fundamental difference between a liquid and a gas which is preserved even above the classical critical point and that 
there is a very definite phase transition line separating them. Assuming such a possibility, the phase diagram in the $P-T$ plane might look as in Fig. 1, where a dotted line separating the liquid from the gas above the classical critical point is indicated as an extension of the usual liquid-gas transition curve. There would, of course, be no latent heat or change in volume in crossing the extended curve, because one can "go around" the classical critical point without observing any discontinuities in these quantities. The transition would have to be of higher order [1] with perhaps anomalies appearing in the isothermal compressibility $K_{T}$, the specific heat at constant pressure $C_{P}$ and the thermal expansion coefficient $\alpha$. The idea that a transition line of higher order exists separating two distinct fluid phases of a single-component system should not be a strange one, for liquid helium is such a system; the superfluid phase is separated from the normal fluid phase by the so-called $\lambda$-line.

We now show that the liquid-gas transition curve can be extended through the classical critical point, at least from an operational point of view. Many investigators have made measurements on many simple systems at and above the classical critical point. Among the few who took proper precautions and made corrections for gravitational effects, the most precise measurements appear to be those of Habgood and Schneider [2] on Xenon. Pressure-density isotherms as well as $P-T$ isochores were taken at a series of temperatures at and above the critical point. From these (smoothed) data, we have computed $K_{T}$, which is shown in Fig. 2. Curve $a$, the

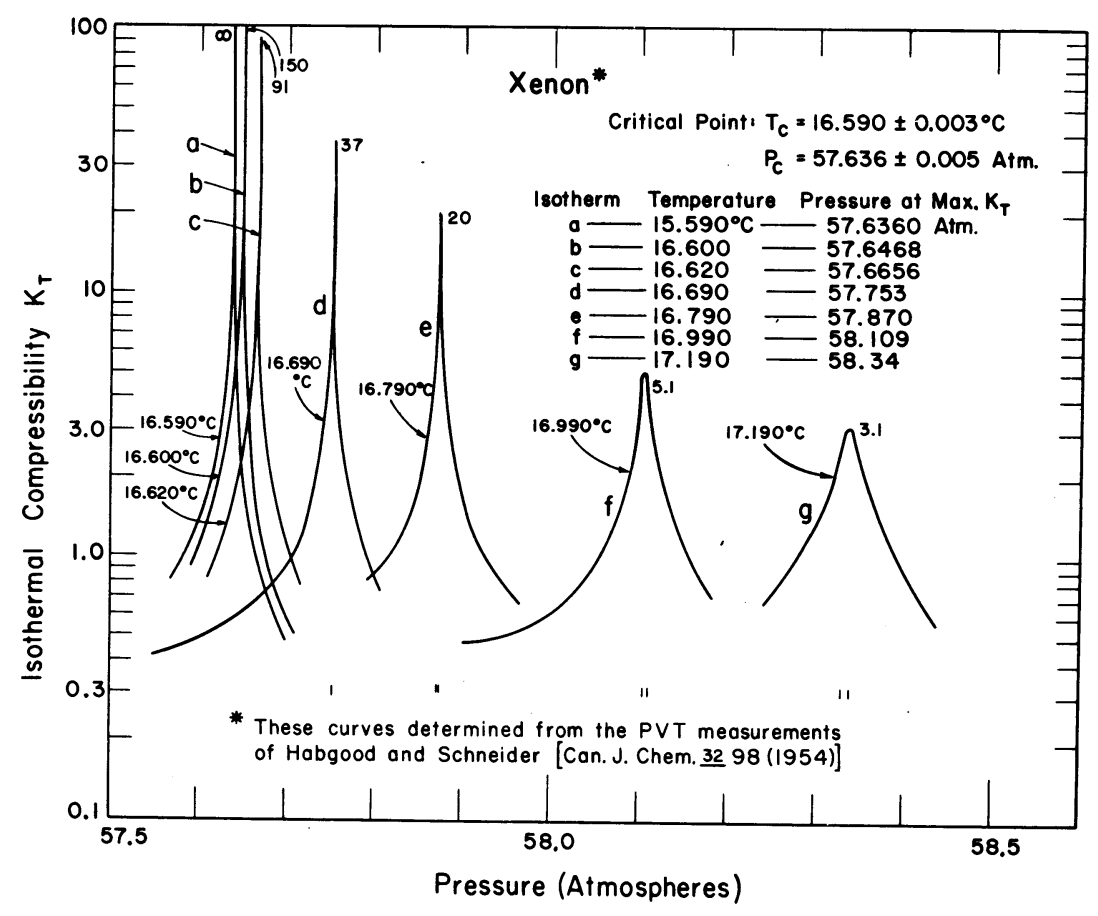

FIGURE 2

Compressability of Xenon (computed from the original data of Habgood and Schneider).

critical isotherm, has a very sharp maximum in $K_{T}$ which appears to be infinite, as expected. The next two curves $(b$ and $c$ ) were computed from isothermal data above the critical point and they also show very sharp maxima, which in these cases are apparently finite. Curves $d, e, f$, and $g$ were computed from isotherms constructed from isochore data, a process which degrades the 
accuracy somewhat. The pressure corresponding to the maximum in $K_{T}$ can be determined very accurately for each isotherm. The resulting points obtained in this way define a curve in the $P-T$ plane and are plotted in Fig. 3, along with measurements of the liquid-gas equilibrium curve from the same experiments [3]. One finds the remarkable result that this curve apparently joins continuously with no change in slope onto the liquid-gas equilibrium curve at the classical critical point [4] (in fact, the "curve" is a straight line over the pressure and temperature range of Fig. 3).

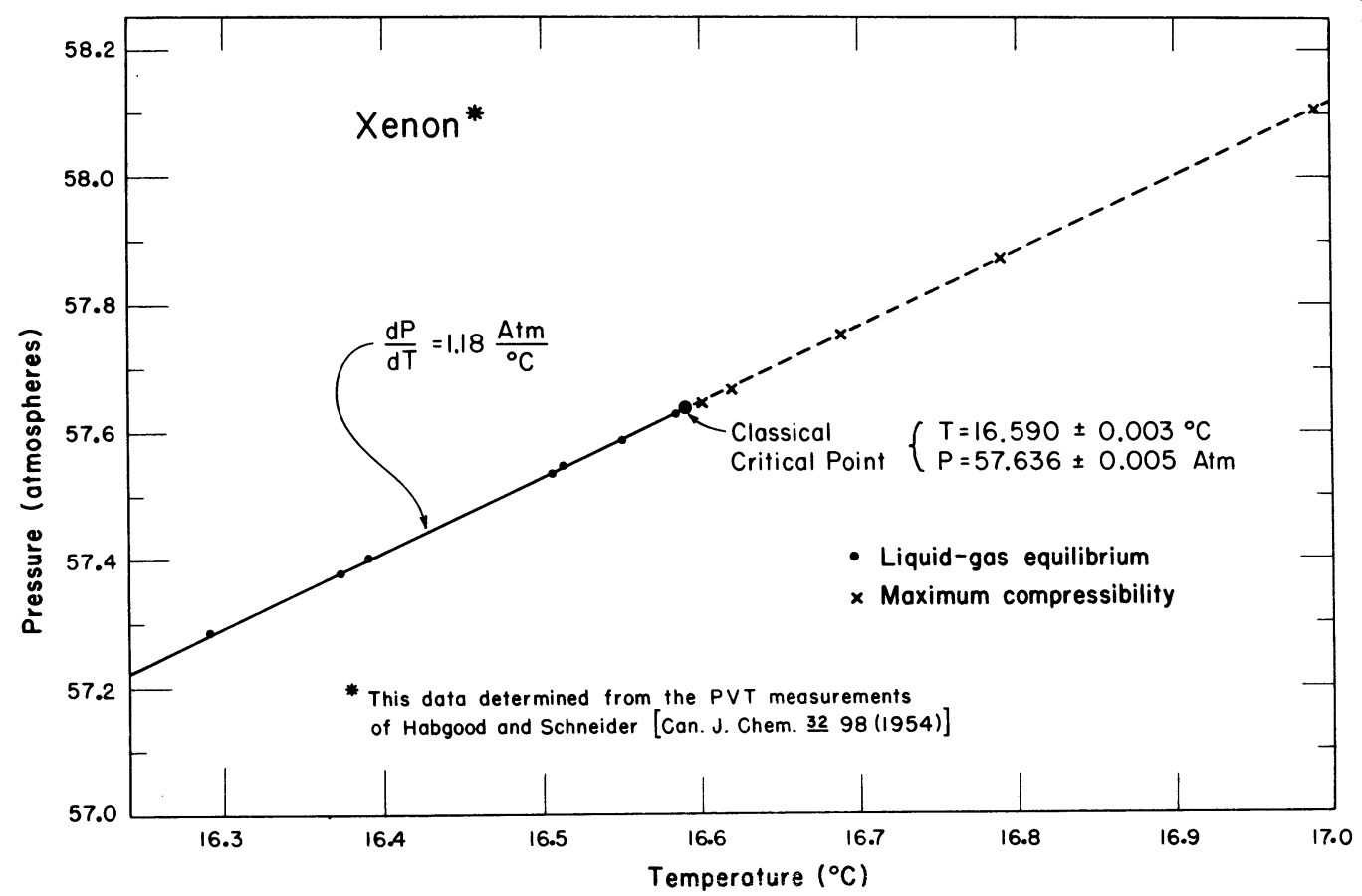

FIGURE 3

P-T diagram of Xenon in the neighborhood of the classical critical point. (Computed from the original data of Habgood and Schneider.)

of what significance is this well defined line and how should it be interpreted? If the compressibility were to become infinite or to undergo a jump in crossing this line, then such nonanalytic behavior would imply the existence of two distinct phases. The data of Habgood and Schneider do not preclude such possibilities [5].

Assuming that the compressibility $K_{T}$ has an infinite maximum along each isotherm, then the meaning of the extension of the liquid-gas equilibrium curve in Fig. 3 would be clear. It would represent the transition line between two distinct fluid phases. This follows from Tisza's considerations concerning the question of critical equilibrium [6]. [Critical equilibrium is defined to be a stable point for which any of the compliance coefficients (i.e. $C_{P}, K_{T}, \alpha$ ) or the specific heat at constant volume $C_{V}$ or both become infinite [7].] His results are presented in the form of a phase rule, which for this particular case states that if a line of critical points exists in a single component system, then that line separates two distinct phases and these two distinct phases differ in some symmetry property but not in the entropy or density [8]. Transitions involving only discontinuities in the compliance coefficients [9] also imply the existence of two distinct phases [10]. It is obvious that these two distinct fluid phases 
above the critical point would be the liquid phase and the gas phase. However, it is not obvious at all in what way the symmetry of the liquid phase would differ from the symmetry of the gas phase. Thermodynamic experiments, of course, can yield no information concerning the symmetry of different phases; this information must come from other types of experiments. One can only speculate as to possible changes in symmetry $[8,11]$.

Regardless of what the change in symmetry might be, one can, according to Landau [10], characterize this change in symmetry by an order parameter $\eta$. In fact, Landau has considered theoretically the case of a higher order transition line meeting a first order line at a critical point. We will now discuss briefly the liquid-gas phase transition above and below the classical critical point in terms of an assumed order parameter. First, one would expect the liquid state to possess the order parameter (i.e., to have lower symmetry than the gas) and that its chemical potential could initially be expressed in terms of $T, P$, and $\eta$.

$$
\mu_{L}=\mu_{L}(T, P, \eta)
$$

The equilibrium value of the order parameter $\eta_{e}$ is determined by that value of $\eta$ which makes $\mu$ a minimum; it is obtained by solving the equation

$$
\frac{\partial \mu_{L}(T, P, \eta)}{\partial \eta}=0
$$

for $\eta(T, P)$. The chemical potential of the liquid expressed only in terms of $T$ and $P$ is

$$
\mu_{L}\left(T, P, \eta_{e}(T, P)\right)
$$

The first order transition line below the classical critical point (point $C$ in Fig. 1) is determined by the usual condition.

$$
\mu_{L}\left(T, P, \eta_{e}(T, P)\right)=\mu_{G}(T, P)
$$

Note that $\eta_{e}(T, P)$ is not equal to zero along the transition line. (The fact that the entropy and volume change discontinuously in crossing the first order curve demand that $\eta_{e}(T, P)$. change from some finite value on the liquid side to 0 on the gas side.) The line of critical points which includes the classical critical point and the points on the line above it is determined from the equation

$$
\eta_{e}(T, P)=0
$$

Thus above the classical critical point the order parameter goes continuously to zero in the liquid state as the critical line is approached, yielding a transition with no latent heat or volume change.

Another interesting property of this system is that $d P / d T$ along the transition curve is continuous at the classical critical point. Above the classical critical point, the slope of the line is determined from the condition $d \eta_{e}(T, P)=0$ and is

$$
\frac{d P}{d T}=-\left(\frac{\partial \eta_{e}}{\partial T}\right)_{T, \eta=0} /\left(\frac{\partial \eta_{e}}{\partial P}\right)_{T, \eta=0}
$$


Below the classical critical point the slope of the vapor pressure curve is given by the Clausius-Clapeyron equation

$$
\frac{d P}{d T}=\frac{S_{G}-S_{L}^{\prime}}{V_{G}-V_{L}}
$$

As the classical critical point is approached, the right-hand side of (7) assumes the indeterminate form $0 / 0$; however, application of L'Hospital's rule shows that (7) is identical to $(6)$ in the limit [12]. The data of Fig. 3 are in very good agreement with this result.

If $K_{T}$ is infinite (and presumably $C_{P}$ and $\alpha$ ) along the extended curve, then one can also discuss this line in terms of the "Pippard relations" [13]. These have been generalized by Tisza [6] and by Buckingham and Fairbank [14]. In the notation of Buckingham and Fairbank they are

$$
\begin{gathered}
C_{P}=T V \alpha\left(\frac{d P}{d T}\right)_{t}+C_{t} \\
\alpha=K_{T}\left(\frac{d P}{d T}\right)_{t}+\alpha_{t}
\end{gathered}
$$

where the concept of a local temperature $t$ is introduced in such a way that $t$ equal to a constant describes a line parallel to the line of critical points ( $t=0$ obviously is the transition line itself). The parameter $C_{t}$ and $\alpha_{t}$ are the "specific heat" and "expansion coefficient" taken in the direction parallel to the transition line; these were assumed by Pippard to be constants (which is a reasonable assumption along the $\lambda$-line of liquid helium). The specific heat at constant volume can be expressed in terms of $C_{t}$ and $\alpha_{t}$

$$
C_{V}=C_{t}-T V\left(\frac{d P}{d T}\right)_{t} \alpha_{t}
$$

If the recent experimental measurements [15] suggesting that $C_{v}$ shows a logarithmic infinity as the classical critical point is approached are correct, then $C_{t}$ (and probably $\alpha_{T}$ if $d P / d T$ is a constant as appears to be the case) must also diverge and this divergence will be at least as strong as a logarithm [16]. Thus along the extended curve the system would probably have the property that $K_{T}, C_{P}$ and $\alpha$ are infinite at all points on the line with $C_{V}$ finite. The classical critical point, however, would be signaled by $C_{V}$ becoming infinite as it is approached [16].

One question concerning the possible extension of the liquid-gas transition curve arises. If the liquid and gas phases have different symmetries then the extension of the transition line cannot end at a point. It must never end or it must meet some other line. It is conceivable that this line might bend upward and meet one of the high pressure solid phases. A more likely probability, however, is the thermal ionization curve.

Needless to say, measurements of any of the compliance coefficients made with resolution sufficient to prove or disprove either a discontinuity or an infinite divergence would be desirable.

This work was partially supported by the Army Research Office (Durham) and the National Aeronautics and Space Administration. The author would also like to acknowledge the use of the general research facilities provided by the National Science Foundation, the Atomic Energy 
Commision and the Advanced Research Projects Agency and Stuart Rice.

\section{REFERENCES and BIBLIOGRAPHICAL NOTES}

1. In the present note, the term "higher order transition" only means a transition that does not have a latent heat or volume change.

2. H. W. HABGOOD and W.G. SCHNEIDER, Can, J. Chem. 32, 98 (1954).

3. The extension of the vapor-pressure curve has been considered before. The first consideration appears to have been by TRAUTZ and ADER [Physik. Zeitschr. 35, 446 (1934] and the next was by EUCKEN [Physik. Zeitschr. 35, 954 (1934)]. Trautz and Ader noted for $\mathrm{CO}_{2}$ that the $P$ - $T$ curve for the critical volume joined onto the vapor-pressure curve continuously. Eucken made measurements of the isothermal Joule-Thomson coefficient $(\partial H / \partial P)_{T}$, where $H$ is the enthalpy) above the critical point of methane. He observed that this coefficient went through a maximum at a certain pressure. This allowed him to construct a curve in the $P-T$ plane which joined onto the liquid-gas transition curve. He pointed out that this curve was different from the critical volume curve of Trautz and Ader. Eucken's curve is probably equivalent to ours since $(\partial H / \partial P)_{T}$ varies like the thermal expansion coefficient [i.e. $(\partial H / \partial P)_{T}=V(1-T \alpha)$ where $V$ is the specific volume] and one expects $\alpha$ and $K_{T}$ (and $C_{P}$ ) to have similar anomalies. Eucken presented a brief analysis of his curves in which he suggested that the transition was third order according to the Ehrenfest scheme.

4. One should note that even though the temperature of the last isotherm in Fig. 2 is only $1.6^{\circ} \mathrm{C}$ above $T_{c}$, this temperature difference is $10^{3}$ times the resolution. Also Eucken [3] followed the maximum in $(\partial H / \partial P)_{T}$ up to about 2 times the critical temperature.

5. An examination of the original data shows that the pressure resolution becomes progressively worse at the higher temperatures, which is probably due to the fact that the data points were not taken as close together as those near the critical point. (An estimate of the pressure resolution is indicated by the separation of two vertical marks under the curves in Fig. 2.) The net effect of such a loss in resolution on a narrow maximum would be to broaden it out and to lower its value. Thus the apparent maximum is not too meaningful because the "actual" maximum could be much larger, perhaps even infite. More meaningful would be a study of the nature of the curve on the "wings" and the approach to the maximum. Because $K_{T}$ must become infinite at the critical point on the critical isotherm, one possible approach to infinity is via a power law $K_{T} \infty\left|P-P_{C}\right|^{\gamma}$. One finds, indeed, that the critical isotherm can be fitted with sucn a power law with $\gamma=-0.72 \pm 0.07$, which may be compared with the value $-2 / 3$ predicted by molecular-field type theories. The curves for the higher temperatures all follow the same general behavior. Away from $P_{c}, K_{T}$ is parallel to the curve for the critical isotherm and as $\left|P-P_{C}\right|$ becomes smaller, $K_{T}$ breaks away from the critical isotherm and approaches a constant. The value of $P-P_{c}$ at which the break occurs, appears related to the resolution. This behavior is suggestive of an infinite maximum which has been broadened by some mechanism.

6. LaSZlo tisza, Ann. Phys. 13, 1 (1961).

7. More specifically, the definition of a critical point is that it is a point where the determinant of the compliance matrix is zero. However, this determinant for a single-component system is equal to $T / V C_{V} K_{T}$, so that the conditions given in the text are qquivalent. 
8. In addition to the well known $\lambda$-line in liquid helium, there exist many examples in solids. A familiar one is the phase transition in quartz at high temperatures; in crossing the transition line there is no latent heat and $C_{P}$ appears to become infinite, thus satisfying the criterion for a critical line. Above this line, quartz exists in a hexagonal crystal structure; as the system is brought through the transition there is an abrupt change in symmetry to a trigonal structure. It is a further result of Tisza's theory that the "unsymmetric" phase should have a multiplicity, which for quartz corresponds to the well known fact that the trigonal state exists in two thermodynamically degenerate modifications - "left" quartz and "right" quartz.

9. A superconductor in the absence of a magnetic field shows only a finite jump in specific heat at the critical temperature. In addition, it should be noted that a superconductor in a magnetic field is an example of a system having a critical point similar to that which we are considering in this paper. On the low temperature side (more precisely, in the region where the penetration depth $\lambda(T)$ is smaller than the characteristic dimension $D$ of the superconductor) the phase transition is first order showing a latent heat. on the high temperature side (where $\lambda(T)>D$ ), the transition is higher order with only a jump in the specific heat.

10. L. LANDAU, Phys. Z. Sowjet. Union 11, 26 and 545 (1937). See also LANDAU and LIFSHITZ Statistical Physics (Addison-Wesley 1958).

11. It should be recalled that the nature of the difference between the two distinct liquid states of helium remained a mystery a good many years after the $\lambda$-line was discovered.

12. The proof of this relation makes no assumption concerning the possible expansion of $\mu$ in a power series in $\eta$. Landau's proof is expressed in terms of the parameters of such an expansion, the validity of which in the neighborhood of phase transitions is always subject to some doubt.

13. A.B. PIPPARD, The Elements of Classical Thermodynanics. (Cambridge University Press, 1957).

14. M.J. BUCKInghaM and W.M. FAIRBANK, Progress in Low Temperature Physics. C.J. Gorter, Editor, (North-Hol land Publishing Co. 1961). Chapter 3.

15. M. I. BAGATSKII, A.V. VORONEL', and V.G. GUSAK, Zh. Eksperim. $i$ Teor. Fiz. 43, 728 (1962) [translation: Sov. Phys. - JETP 16, 517 (1963)]; A.V. VORONEL', Ya.R. CHASHKIN, V.A. POPOV, and V.G. SIMKIN, Zh. Eksperim. i Teor. Fiz. 45, 828 (1963) [translation: Sov. Phys. - JETP 18, $568(1964)$ ]. C.E. CHASE, R. C. WILLIAMSON and LASZLO TISZA, Phys. Rev. Lett. 13, 467 (1964).

16. We have succeeded in deriving equations (9) and (10) by approaching the classical critical point from the 2-phase region. An additional result that comes out of this derivation is that $C_{t}{ }^{2} / C_{p}$ and $\alpha_{t}{ }^{2} / \alpha$ should approach zero as the classical critical point is approached. If the molecular field type behavior [5] is assumed with $\gamma=-2 / 3$, then this would

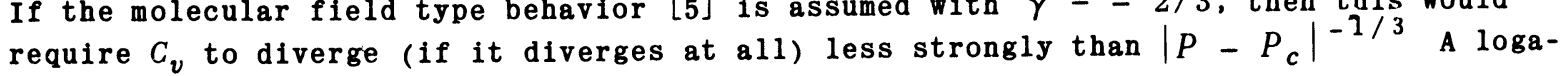
rithmic divergence is certainly consistent with this requirement. 